\section{(A) Check for updates}

Cite this: Dalton Trans., 2021, 50 8258

DOI: $10.1039 / \mathrm{d} 1 \mathrm{dt} 90084 \mathrm{f}$

rsc.li/dalton

\title{
Correction: Axially coordinated chiral salen Mn(III) anchored onto azole onium modified ZnPS-PVPA as effective catalysts for asymmetric epoxidation of unfunctionalized olefins
}

\author{
Jing Huang, Xiangkai Fu, ${ }^{\star}$ Gang Wang, Qiang Miao and Guomin Wang
}

Correction for 'Axially coordinated chiral salen Mn(III) anchored onto azole onium modified ZnPS-PVPA as effective catalysts for asymmetric epoxidation of unfunctionalized olefins' by Jing Huang et al., Dalton Trans., 2012, 41, 10661-10669, DOI: 10.1039/C2DT30081H.

The authors regret sections of unattributed text overlap throughout this Dalton Transactions article with their previous work, primarily an Applied Catalysis A: General article which was not cited. ${ }^{1}$ There are significant portions of text overlap with this Applied Catalysis A: General article, particularly in the Introduction and Experimental sections and in sections 3.1.8.2 and 3.3-3.4 in the Results and discussion but there are also sections of original work.

The authors wish to clarify the differences between this Dalton Transactions article and their previous work in Applied Catalysis A: General ${ }^{1}$ where an azole onium linker is used in the catalyst in this Dalton Transactions article and an aryldiamine linker is used in the catalyst in the Applied Catalysis A: General paper. In addition, the mechanisms of the two catalytic systems are different. In the Applied Catalysis A: General paper, the bulkier linker benzidine makes it difficult for the substrates to approach the catalyst and the rigid linker increases the ee values. The steric properties of the linkages have a vital impact on the configuration of the transition state for the asymmetric reactions. The additives played such different roles that the catalytic activities decreased with the addition of NMO when $m$-CPBA was the oxidative system or slightly increased in the presence of imidazole with $\mathrm{NaIO}_{4}$ as the oxidative system. In the Dalton Transactions article, as the structures of imidazole and triazole onium in the heterogeneous catalysts are similar to NMO, and due to the steric hindrance from $m$-CPBA, the optimal geometric configuration of the reactive intermediate salen $\mathrm{Mn}(\mathrm{v})=\mathrm{O}$ and its transition state altered when $N$-oxide ligands were added in the $m$-CPBA oxidative system. However, when using the $\mathrm{NaIO}_{4}$ oxidative system, the steric hindrance from $\mathrm{NaIO}_{4}$ was negligible relative to the $m$-СРВA oxidative system such that the catalytic activity and enantioselectivity were not influenced when the imidazole additive was added to the mixture.

The authors also regret that it was not clear in the original article that Fig. S6, S7 and S9 in the ESI had been reproduced from their related Applied Catalysis A: General paper. ${ }^{1}$

The Royal Society of Chemistry apologises for these errors and any consequent inconvenience to authors and readers.

\section{References}

1 J. Huang, X. Fu and Q. Miao, Appl. Catal., A, 2011, 407, 163-172.

College of Chemistry and Chemical Engineering Southwest University, Research Institute of Applied Chemistry Southwest University, The Key Laboratory of Applied Chemistry of Chongqing Municipality, The Key Laboratory of Eco-environments in Three Gorges Reservoir Region Ministry of Education, Chongqing 400715, China.

E-mail: hj41012@163.com; Fax:+86 2368254000; Tel: +86 2368253704 